Supporting Information

\title{
Modeling Multicomponent Gas Adsorption in Nanoporous Materials with Two Versions of Nonlocal Classical Density Functional Theory

\author{
Musen $\mathrm{Zhou}^{1 \dagger}$, Jingqi Wang ${ }^{2 \dagger}$, Jose Garcia ${ }^{1}, \mathrm{Yu} \mathrm{Liu}^{3 *}$ and Jianzhong $\mathrm{Wu}^{1 *}$ \\ ${ }^{1}$ Department of Chemical and Environmental Engineering, University of California, Riverside, \\ CA 92521, United States \\ ${ }^{2}$ Department of Chemical Engineering, Tsinghua University, Beijing, 100084, China \\ ${ }^{3}$ School of Chemical Engineering and Technology, Sun Yat-sen University, Zhuhai, Guangdong \\ 519000, China
}

This supporting information contains the computational details of grand canonical Monte Carlo (GCMC) simulation and classical density functional theory (cDFT) for predicting multicomponent gas adsorption. It also includes adsorption isotherms and selectivity for $\mathrm{CO}_{2} / \mathrm{CH}_{4}$, additional density isosurfaces for $\mathrm{CH}_{4} / \mathrm{CO}_{2} / \mathrm{H}_{2}$ in MOF-5 at $313.15 \mathrm{~K}$ and 100 bar and computational expense comparison for ternary mixture.

\section{Computational Details}

Table S1 lists the Lennard-Jones (LJ) parameters for gas molecules considered in this work. ${ }^{1-}$ 5 The grid size in all cDFT calculations is $0.5 \AA$. The potential is truncated and shifted to zero at $12.9 \AA$. One unit cell of MOF-5 with periodic boundary condition is used in the calculation. CG_DESCNT package is used to minimize the grand potential with the convergence criteria set as the gradient to be $1 \times 10^{-3}$.

\footnotetext{
$\uparrow$ These authors contributed equally.

*To whom correspondence should be addressed. Email: jwu@engr.ucr.edu or liuyu89@mail.sysu.edu.cn
} 
In this work, GCMC simulations are all carried with the RASPA package. ${ }^{7}$ For adsorption at extremely low pressure where single component adsorption, 5 million steps are used for both equilibration and production. For adsorption at high pressure, 500,000 steps are used for the equilibration and production. When comparing the computational cost with cDFT, 50,000 steps are used for the equilibration and production in GCMC.

Table S1. The Lennard-Jones parameters for gas compounds considered in this work

\begin{tabular}{ccc}
\hline adsorbate & $\varepsilon / k_{B}(\mathrm{~K})$ & $\sigma(\AA)$ \\
\hline $\mathrm{CO}_{2}$ & 236.1 & 3.75 \\
$\mathrm{CH}_{4}$ & 148 & 3.73 \\
$\mathrm{H}_{2}$ & 34.2 & 2.96 \\
$\mathrm{Ar}$ & 119.5 & 3.41 \\
$\mathrm{Kr}$ & 165.2 & 3.66 \\
\hline
\end{tabular}

\section{Adsorption Isotherm and Selectivity}

Figure S1 shows the adsorption isotherms of all one-component gas adsorptions from GCMC.

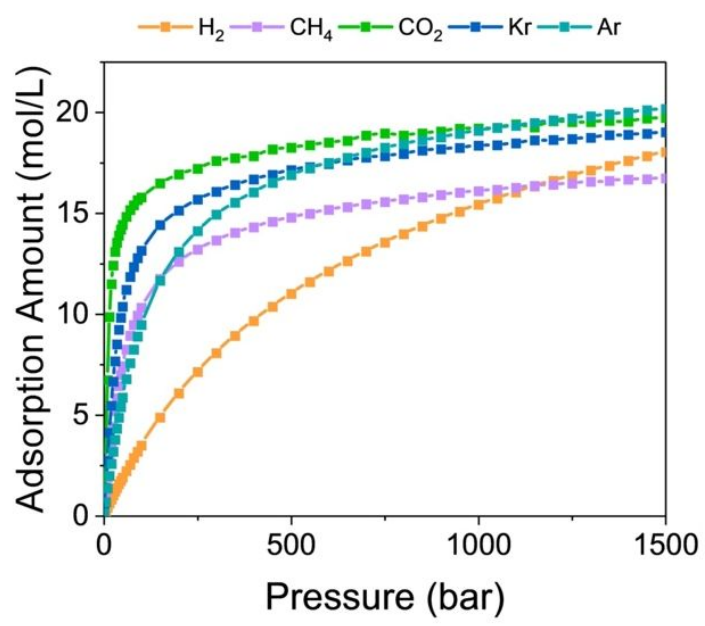

Figure S1. Adsorption isotherm for individual gases calculated from GCMC in MOF-5 for $\mathrm{Kr}$ and $\mathrm{Ar}$ at $297 \mathrm{~K}$ and $\mathrm{H}_{2}, \mathrm{CH}_{4}, \mathrm{CO}_{2}$ at $313.15 \mathrm{~K}$.

In addition to gas mixtures discussed in the main text, we have simulated an equimolar binary gas mixture of $\mathrm{CH}_{4} / \mathrm{CO}_{2}$. The adsorption isotherms and selectivity of $\mathrm{CH}_{4} / \mathrm{CO}_{2}$ at low to 
moderate pressure are shown in Figure S2. At low pressure, both mixture cDFT and IAST can quantitively predict the adsorption isotherms compared to the GCMC results. At infinite dilution (extremely low loadings), the selectivity predicted by IAST also converges to the ideal limit because of the high accuracy in single-component adsorption isotherm.

- $\mathrm{CH}_{4}(\mathrm{GCMC})-\mathrm{CH}_{4}$ (CDFT-WDA) $\cdots \cdots \cdots \cdot \mathrm{CH}_{4}$ (CDFT-MFA) -.-- $\mathrm{CH}_{4}$ (IAST)

- $\mathrm{CO}_{2}(\mathrm{GCMC})-\mathrm{CO}_{2}$ (CDFT-WDA) …...... $\mathrm{CO}_{2}$ (CDFT-MFA) -..- $\mathrm{CO}_{2}$ (IAST)
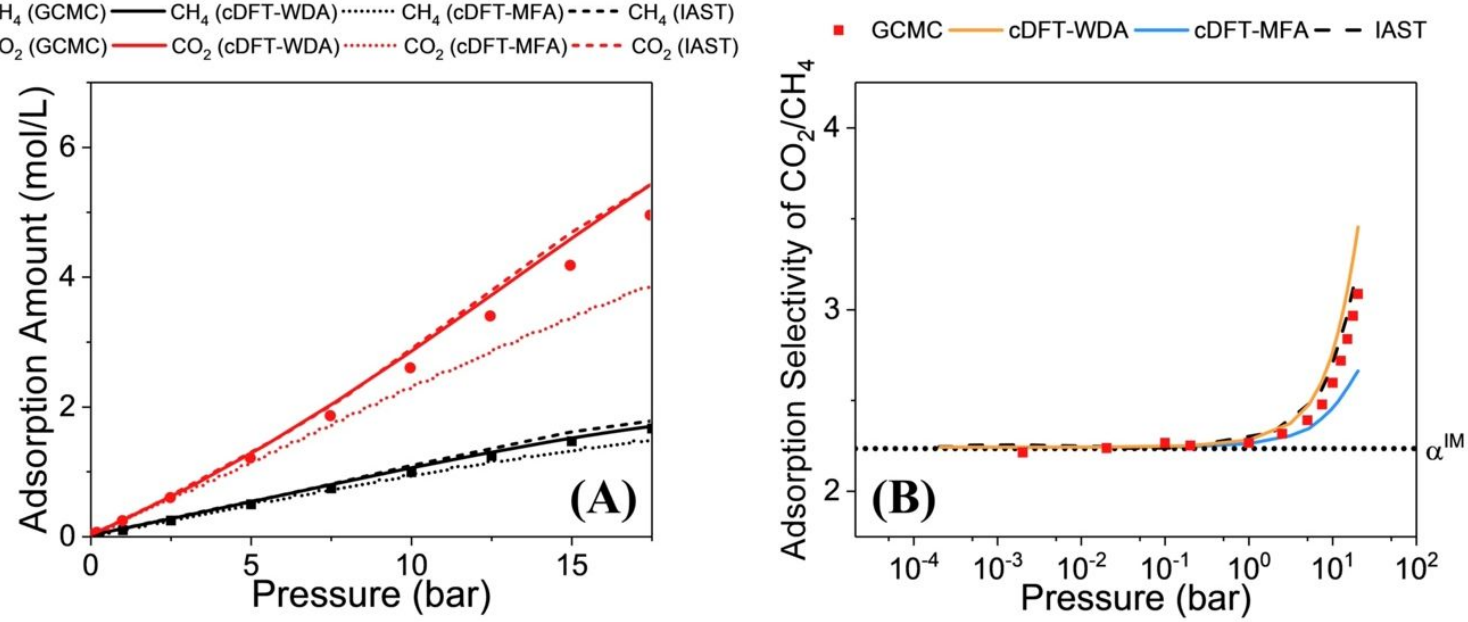

Figure S2. Adsorption isotherms (A) and selectivity (B) for an equimolar mixture of $\mathrm{CH}_{4}$ and $\mathrm{CO}_{2}$ in MOF-5 at $313.15 \mathrm{~K}$ up to 15 bar. The dotted line represents the adsorption selectivity at infinite dilution. 

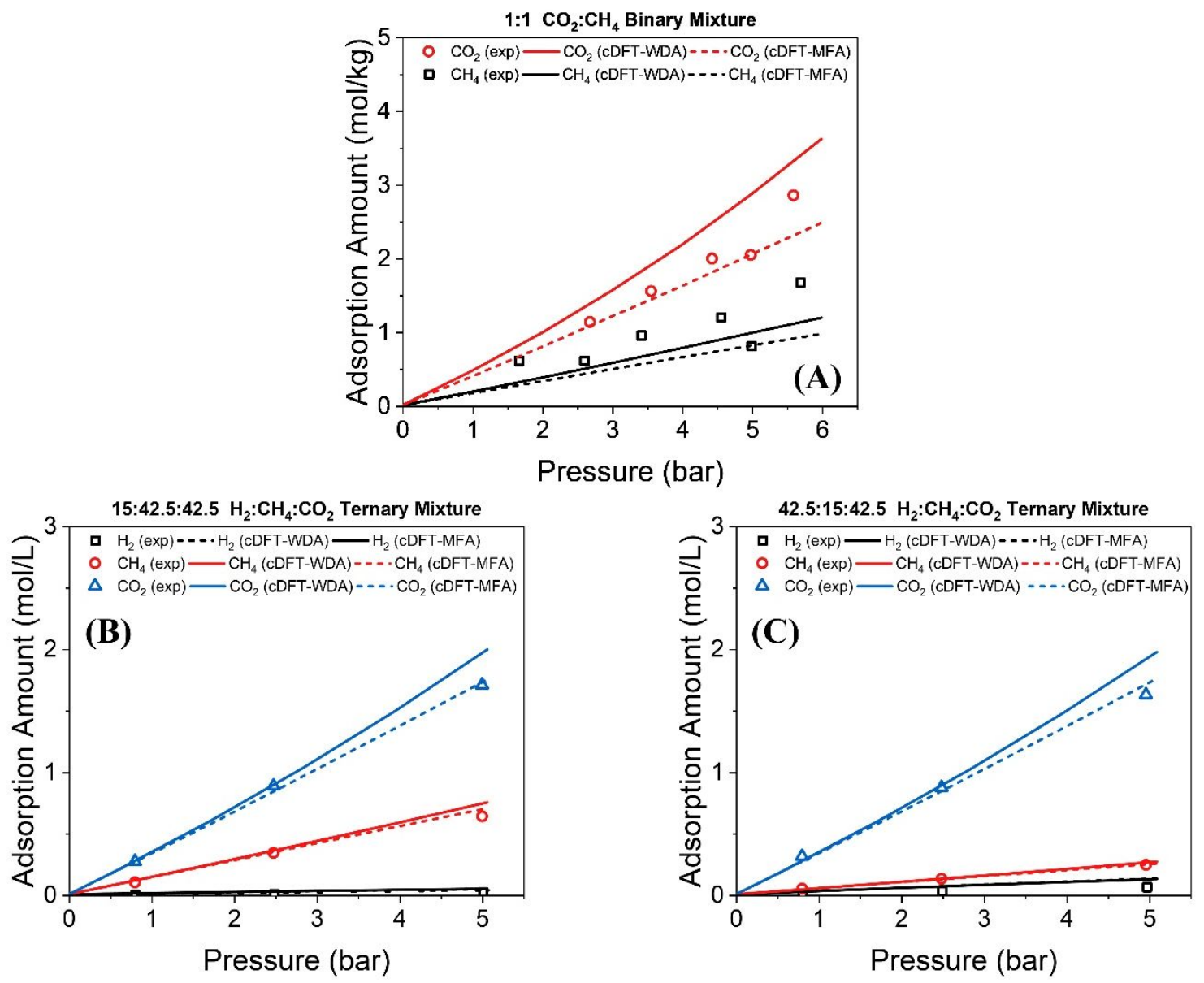

Figure S3. Adsorption isotherms for (A) equimolar mixture of $\mathrm{CO}_{2}$ and $\mathrm{CH}_{4}$ in MOF-5 at $297 \mathrm{~K}$ and ternary mixture of $\mathrm{H}_{2}, \mathrm{CH}_{4}$ and $\mathrm{CO}_{2}$ in MOF-5 at $297 \mathrm{~K}$ with different bulk concentration: (B) $\mathrm{H}_{2}: \mathrm{CH}_{4}: \mathrm{CO}_{2}=15: 42.5: 42.5$ (C) $\mathrm{H}_{2}: \mathrm{CH}_{4}: \mathrm{CO}_{2}=42.5: 15: 42.5$. The dashed lines are calculated from the classical density functional theory with mean field approximation (cDFT-MFA), the solid lines are from the classical density functional theory with weighted density approximation (cDFT-WDA) and the symbol are experimental measurement from literature ${ }^{8-9}$.

Figure S4 shows the adsorption isotherms and selectivity of $\mathrm{CH}_{4} / \mathrm{CO}_{2}$ at pressure up to 450 bar. Similar to the ternary system discussed in the main text, IAST fails to predict the competitive adsorption of $\mathrm{CO}_{2}$ in MOF-5 at high pressure. 
- $\mathrm{CH}_{4}$ (GCMC) $-\mathrm{CH}_{4}$ (CDFT-WDA) …….... $\mathrm{CH}_{4}$ (CDFT-MFA) - - - $\mathrm{CH}_{4}$ (IAST)

- $\mathrm{CO}_{2}(\mathrm{GCMC})-\mathrm{CO}_{2}$ (cDFT-WDA) $\cdots \cdots \cdot \mathrm{CO}_{2}$ (cDFT-MFA)--.- $\mathrm{CO}_{2}$ (IAST)

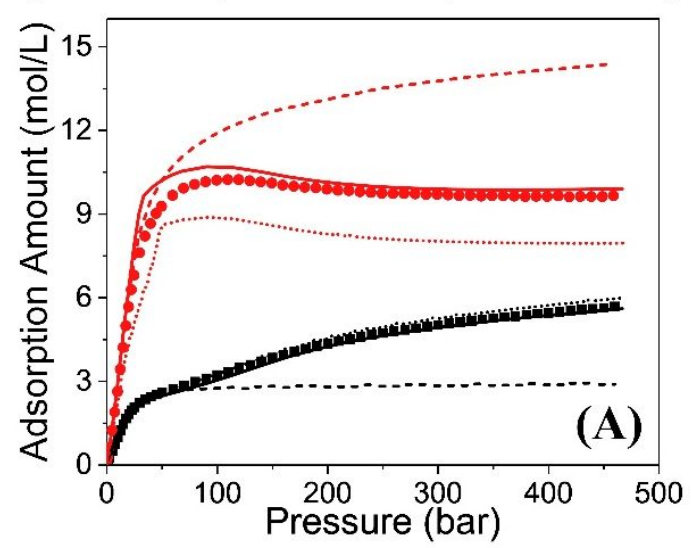

GCMC - CDFT-WDA - CDFT-MFA - - - IAST

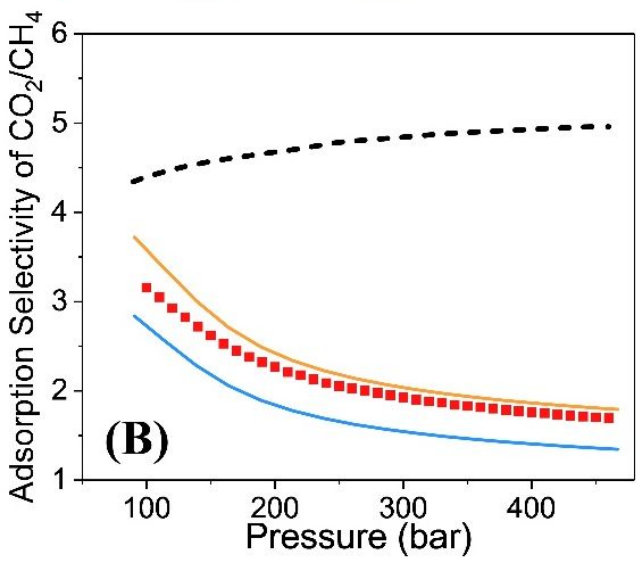

Figure S4. Adsorption isotherms (A) and selectivity (B) for an equimolar mixture of $\mathrm{CH}_{4}$ and $\mathrm{CO}_{2}$ in MOF-5 at $313.15 \mathrm{~K}$ up to 450 bar.

\section{Density Isosurfaces}

Figure $\mathrm{S} 5$ presents the density iso-surfaces of $\mathrm{CH}_{4}, \mathrm{CO}_{2}$ and $\mathrm{H}_{2}$ in a ternary mixture with the bulk molar composition $\left(\mathrm{H}_{2}: \mathrm{CH}_{4}: \mathrm{CO}_{2}=15: 42.5: 42.5\right)$. For all gas compounds, the local density in the iso-surface is set as one half of the maximum value. It shows clearly that hydrogen (yellow iso-surface) is closest to the adsorbent surface (node and linker atoms). By contrast, carbon dioxide (orange isosurface) is furthest away from the adsorbent surface while methane (red isosurface) stays in between hydrogen and carbon dioxide as expected from their molecular sizes.

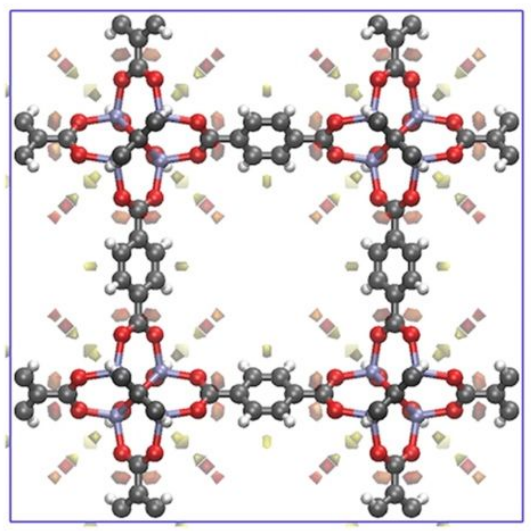


Figure S5. Density iso-surfaces of $\mathrm{CH}_{4}, \mathrm{CO}_{2}$ and $\mathrm{H}_{2}$ for a ternary mixture with bulk composition $\left(\mathrm{H}_{2}: \mathrm{CH}_{4}: \mathrm{CO}_{2}=15: 42.5: 42.5\right)$ in MOF-5 at $313.15 \mathrm{~K}$ and gas pressure 100 bar. The red, orange and yellow colors represent the density iso-surfaces of $\mathrm{CH}_{4}, \mathrm{CO}_{2}$ and $\mathrm{H}_{2}$, respectively. The local density of each iso-surface is taken as half of the maximum local density value. The grey, purple, red and white spheres represent carbon, zinc, oxygen and hydrogen atoms, respectively.

\section{Computational Expense Comparison}

Figure S6 shows the computational time and speedup factor for the adsorption of ternary mixture, $\mathrm{H}_{2} / \mathrm{CO}_{2} / \mathrm{CH}_{4}$, in MOF-5 at $313.15 \mathrm{~K}$.
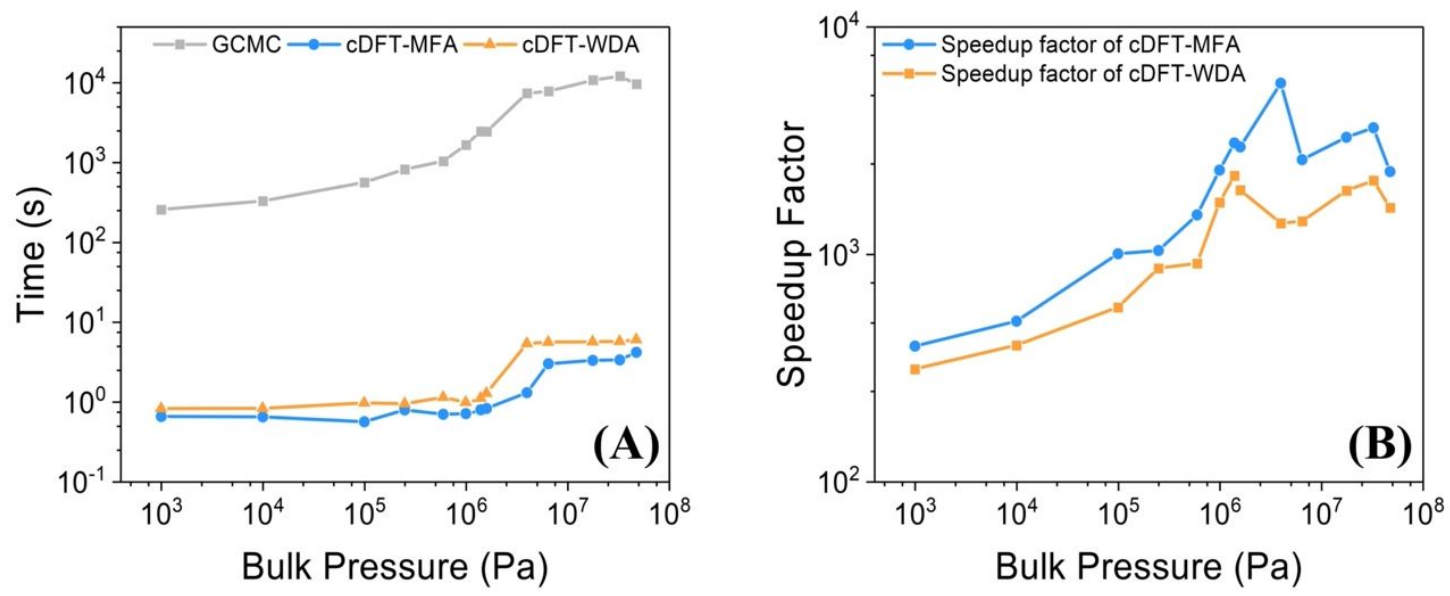

Figure S6. (A) Computation time of GCMC, cDFT-MFA and cDFT-WDA versus system pressure for ternary mixture of $\mathrm{H}_{2}, \mathrm{CH}_{4}$ and $\mathrm{CO}_{2}$. (B) The speedup factors of cDFT-MFA and cDFT-WDA are based on the computation time of GCMC.

\section{Reference}

1. Steele, W. A., The Physical Interaction of Gases with Crystalline Solids: I. Gas-Solid Energies and Properties of Isolated Adsorbed Atoms. Surf Sci 1973, 36, 317-352.

2. Martin, M. G.; Siepmann, J. I., Transferable Potentials for Phase Equilibria. 1. UnitedAtom Description of N-Alkanes. $J$ Phys Chem B 1998, 102, 2569-2577.

3. Buch, V., Path Integral Simulations of Mixedpara-D2andortho-D2clusters: The Orientational Effects. J Chem Phys 1994, 100, 7610-7629.

4. $\quad$ Lastoskie, C.; Gubbins, K. E.; Quirke, N., Pore Size Heterogeneity and the Carbon Slit Pore: A Density Functional Theory Model. Langmuir 1993, 9, 2693-2702. 
5. Pellenq, R. J.; Nicholson, D., Intermolecular Potential Function for the Physical Adsorption of Rare Gases in Silicalite. J Phys Chem 1994, 98, 13339-13349.

6. Hager, W. W.; Zhang, H. C., Algorithm 851: Cg Descent, a Conjugate Gradient Method with Guaranteed Descent. ACM Transactions on Mathematical Software 2006, 32, 113-137.

7. Dubbeldam, D.; Calero, S.; Ellis, D. E.; Snurr, R. Q., Raspa: Molecular Simulation

Software for Adsorption and Diffusion in Flexible Nanoporous Materials. Mol Simul 2016, 42, $81-101$.

8. $\quad$ Kloutse, F.; Hourri, A.; Natarajan, S.; Benard, P.; Chahine, R., Experimental Benchmark Data of Ch4, Co2 and N2 Binary and Ternary Mixtures Adsorption on Mof-5. Sep Purif Technol 2018, 197, 228-236.

9. $\quad$ Kloutse, F.; Hourri, A.; Natarajan, S.; Benard, P.; Chahine, R., Hydrogen Separation by Adsorption: Experiments and Modelling of H2-N2-Co2 and H2-Ch4-Co2 Mixtures Adsorption on Cubtc and Mof-5. Microporous Mesoporous Mater 2018, 271, 175-185. 